solution (2-3 $\mathrm{mol} \%$ ) for about $5 \mathrm{~min}$, followed by immersions in a diluted solution $(0.5-1 \mathrm{~mol} \%)$ for $10 \mathrm{~min}$. In this way, the researchers achieved a 16-layer woodpile PC with an in-plane spacing of $1 \mu \mathrm{m}$ and a layer spacing of $1.2 \mu \mathrm{m}$, built up by smooth rods, $150 \mathrm{~nm}$ in thickness, and excellent mechanical strength. The resolution limit of the direct-write fabrication process and multi-step etching process is beyond the diffraction limit and comparable to the fabrication limit achievable in photosensitive polymers. The researchers attributed this resolution limit to the threshold of the fabrication technique, combined with the multi-step etching process. Due to the ellipsoidal shape of each rod and the geometry of the woodpile structure, no complete bandgap could be achieved, and the researchers only observed partial bandgaps along the stacking direction.

According to calculations produced by the researchers, the fabrication process should produce two pronounced dips in the transmission spectrum, a first-order stop gap at a normalized frequency of $0.28-0.29$ and $74 \%$ suppression ratio in transmission, and a higher order gap at a frequency of $0.40-0.41$ and $25 \%$ suppression ratio. The measured optical response of the woodpile PC is in good agreement with the results of the calculations. Further calculations performed by the researchers revealed that these bandgaps could be engineered and tuned by either changing the filling ratio or the lattice constant of the PCs, which they confirmed experimentally.

The researchers consider that the use of such higher order bandgaps "provide an effective alternative to achieve photonic bandgaps in the telecommunication wavelength region, circumventing the need for structural miniaturization of the PCs."

JOAN J. CARVAJAL

\section{Materials Design Principles from Ancient Armored Fish Give Clues for Improved Engineered \\ Biomimetic Structural Materials}

Dermal armor in fish first appeared 500 million years ago in the Paleozoic period. As ancient fish became more predatory, armor design evolved incorporating smaller plates instead of larger plates and fewer, thinner layers replacing multiple, thick layers resulting in a lighter weight, balancing protection with mobility for maximum survival. B. Bruet, J. Song, M. Boyce, and C. Ortiz of the Massachusetts Institute of Technology have used nanoindentation and finite element analysis simulations to uncover structure-property-function relationships in individual scales of a model species of armored fish, Polypterus senegalus. The materials design principles of P. senegalus fish armor in the context of their primary environmental threat, penetrating bite attacks, and mechanically protective function, could lead to better bioinspired engineered designs for human body armor, according to the researchers.

In the September 2008 issue of Nature Materials (DOI: $10.1038 /$ nmat2231 p. 748), the researchers reported on a multiscale experimental and computational approach to examine multilayering and gradation within individual scales from $P$. senegalus. These freshwater fish, also known as the Gray bichir or Senegal bichir, appeared about 96 million years ago but still retain characteristics of their ancient predecessors. The researchers reported that $P$. senegalus scales consist of four organicinorganic nanocomposite layers: ganoine ( $10 \mu \mathrm{m}$ thick), dentine ( 50 $\mu \mathrm{m}$ thick), isopedine ( $\sim 40 \mu \mathrm{m}$ thick), and a bone basal plate ( 300 $\mu \mathrm{m}$ thick), from the outer to inner surface, respectively. Penetrationresistance and elastic and plastic mechanical properties of the layers were examined spatially on individual scales by nanoindentation.

One overlying mechanical design theme revealed by these experiments was the juxtaposition of multiple distinct reinforcing layers each with its own unique deformation and energy dissipation mechanisms. Multiple cross-sectional indents revealed a decrease in Oliver-Pharr indentation modulus and hardness from outer to inner
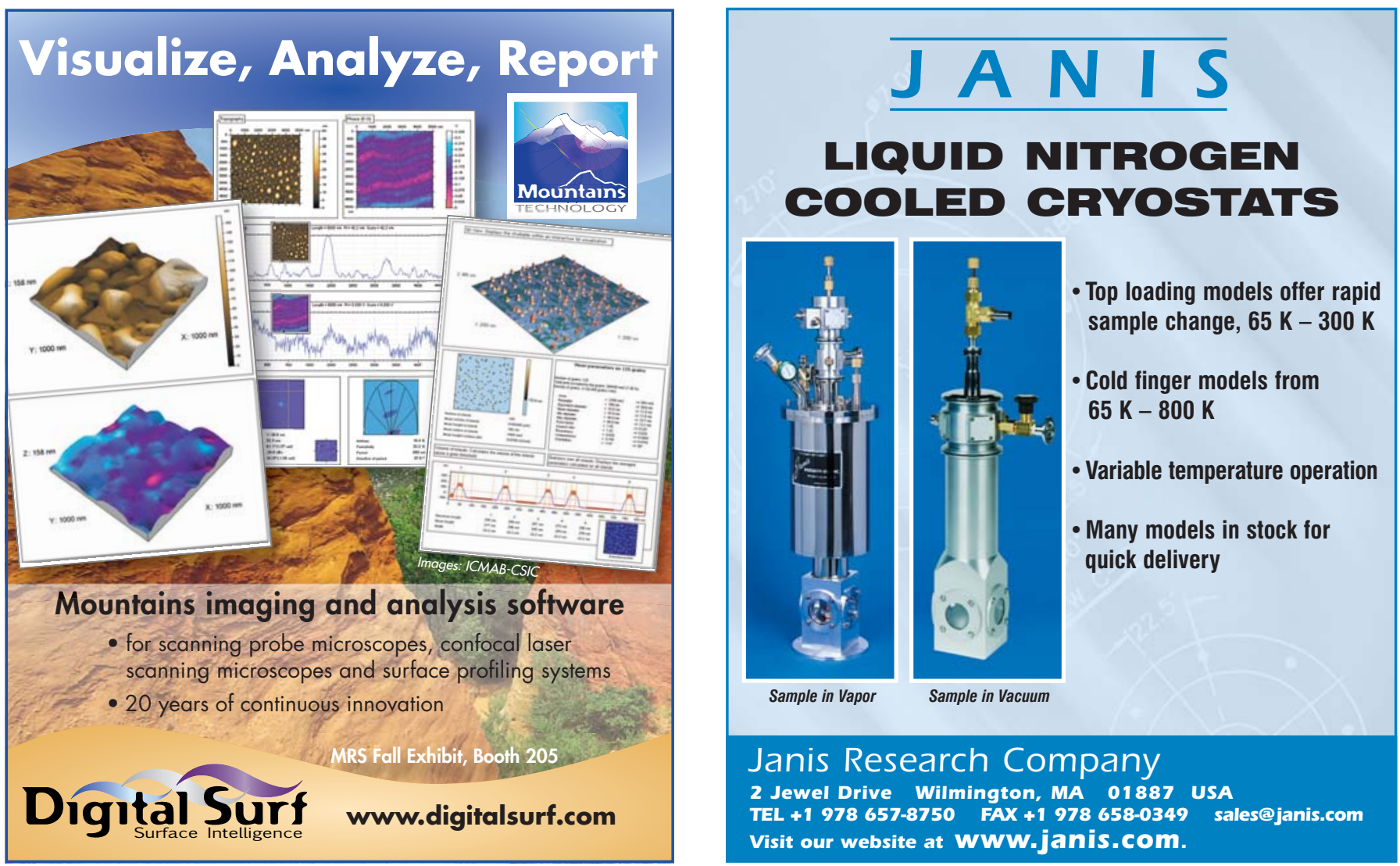
scale surfaces. Observed mechanical properties correlated with the known composition of each layer. The researchers concluded that layer thickness, grading of mechanical properties within and between layers, as well as material layer sequence within individual scales of $P$. senegalus are physiologically important for abating penetration and facilitating protection while yielding biomechanical flexibility and maneuverability of the entire fish. Materials design principles learned from these "living fossils" may hold promise for improved design of engineered biomimetic structural materials, such as protective armor for humans or military vehicle applications, said the researchers.

SAMESHA R. BARNES

\section{Spatial Light Scattering Reveals New Information on Atmospheric Ice Crystals}

Uncertainty about the future of Earth's climate has opened avenues of research into the elements shaping the climate. Meteorological evidence suggests that there are correlations between cloud properties and climate change. Clouds, comprised mainly of water vapor, droplets, and ice crystals, both absorb heat and reflect radiation from the sun. However, the magnitude of their ability to alter climate is unknown. What is known is that the size, shape, and number of particles present in the cloud determines its radiative properties. Currently, analysis of these features is frequently performed by instruments such as the Cloud Particle Imager (CPI), which uses a pulsing laser to capture real images of ice crystals on a CCD camera. However, the CPI is limited to imaging particles greater than $\sim 25 \mu \mathrm{m}$. The use of the device described in this study will allow data on particle size, shape, and quantity to be acquired from particles approaching $1 \mu \mathrm{m}$.

P.H. Kaye and colleagues at the University of Hertfordshire, P.J. DeMott of Colorado State University, and C. Saunders and colleagues at the University of Manchester have used a light scattering approach to analyze atmospheric ice crystals. In the July 1 issue of Optics Letters (DOI:10.1364/OL.33.001545; p. 1545), the researchers described the design and testing of a novel instrument that can capture high-resolution light scattering patterns for sizes well below the resolution of conventional cloud imaging probes. The instrument is composed of a temperaturecontrolled light scattering chamber with a tapered aerosol inlet tube and a vent, an intensified CCD camera, a particle detection trigger photomultiplier detector, a pellicle beam splitter, and a frequencydoubled NdYAG laser. Air flows through the inlet tube at $80 \mathrm{~m} / \mathrm{s}$ and exits into the scattering chamber with a flow diameter of $2.5 \mathrm{~mm}$, where it crosses the laser beam. The pattern of light scattered by individual particles carried in the flow is then captured by the optical system, with a small percentage of light being directed by the pellicle beam splitter onto the detector and the remainder directed to the ICCD camera. Particle size is determined by the magnitude of the signal produced by the photomultiplier detector, and the same signal is used to trigger the ICCD camera to record a high-resolution image of the particle's scattering pattern. This was demonstrated in preliminary experiments, using the University of Manchester Icing Cloud Chamber where ice crystals ranging from $3.5 \mu \mathrm{m}$ to $22 \mu \mathrm{m}$ in diameter were observed for a range of temperatures and humidity. The ice crystals displayed scattering typical of the hexagonal symmetry found in ice. Interpretation of the scattering patterns is required to precisely determine the shape of the crystals. Currently, for less complex crystal shapes, understanding of crystal morphology is achieved by inversion of scattering patterns using theoretical scattering models or by comparison with scattering patterns from known crystal shapes. Future progress in modeling will allow for increased accuracy of the interpretation of scattering patterns for complex crystal shapes. The research in this study was able to uncover information on the morphology and size
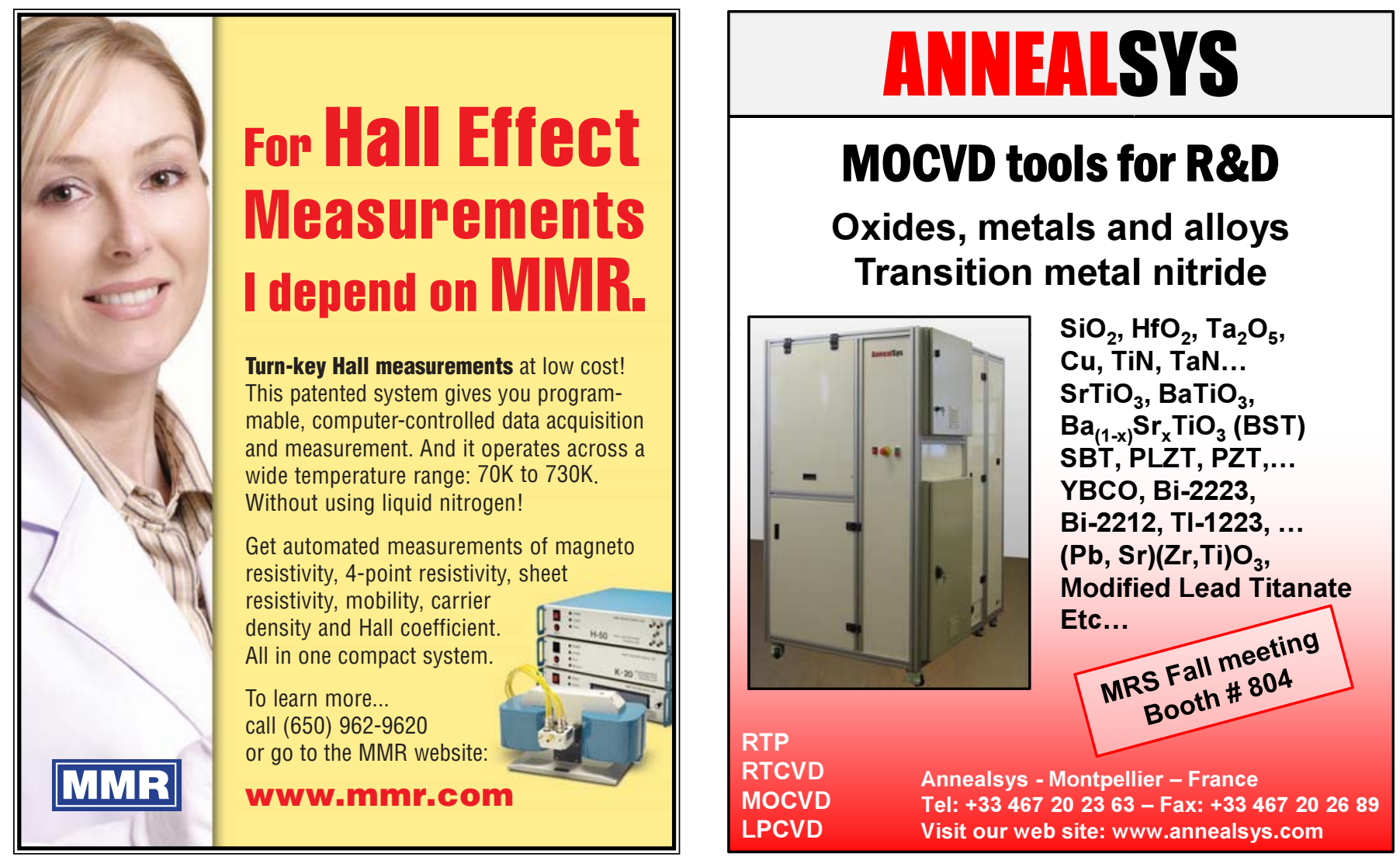